\title{
Combining equal-channel angular extrusion (ECAE) and heat treatment for achieving high strength and moderate ductility in an Al-Cu alloy
}

\author{
Matthias Hockauf ${ }^{1, ~ a ~, ~ L o t h a r ~ W . ~ M e y e r ~}{ }^{1, b}$, Lutz Krüger ${ }^{2, ~ c ~}$ \\ ${ }^{1}$ Chemnitz University of Technology, Institute for Materials and Impact Engineering, Erfenschlager \\ Str. 73, 09125 Chemnitz, Germany \\ ${ }^{2}$ TU Bergakademie Freiberg, Institute for Materials Engineering; Gustav-Zeuner Straße 5; 09596 \\ Freiberg, Germany

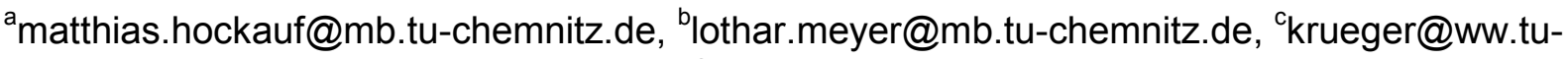 \\ freiberg.de
} Keywords: Severe plastic deformation (SPD), Grain refinement, Aging, Workability, Thermal
stability, Recovery, Annihilation, Precipitation, Backpressure

\begin{abstract}
The effect of equal-channel angular extrusion (ECAE) on mechanical properties of an AA2017 produced by powder metallurgy is investigated. Special attention is given to the influence of heat treatment, processing temperature and backpressure on the workability for achieving high strength and moderate ductility. This is of special interest, since it is often reported that Al-Cu alloys have low ductility and therefore are prone to cracking during severe plastic deformation. It is shown that ECAE at high temperatures $\left(>220^{\circ} \mathrm{C}\right)$ does not necessitate backpressure to ensure homogeneous deformation but leads to a significant sacrifice in strength due to in-situ precipitation. Thus, most of the extrusions are done at considerably low temperatures. Performing room temperature-extrusion is most effective in achieving high strengths but also requires high backpressures. Due to severe strain hardening during processing, the strength increase is combined with a reduction in ductility. Recently it was reported that a post-ECAE aging of pre-ECAE solution treated material is effective in enhancing the ductility of aluminium alloys. This approach was successfully transferred to the current alloy. A high-temperature, short-time aging after only one extrusion, for example, doubles the failure strain to a value of $\sim 13 \%$. Compared to the naturally aged condition with coarse grains that serves as reference (T4), an increase of $15 \%$ in yield stress (YS) was obtained while retaining the ultimate tensile stress (UTS). Another effective approach is the combination of a pre-ECAE solution treatment with subsequent under-aging prior to ECAE. It is shown that performing ECAE at medium temperatures $\left(160-180^{\circ} \mathrm{C}\right)$ enables a better workability and additionally gives higher strengths and better ductility compared to the processing in the water quenched condition. A remarkable YS of $530 \mathrm{MPa}$ and an UTS of $580 \mathrm{MPa}$ combined with a moderate failure strain of $11.6 \%$ were achieved.
\end{abstract}

\section{Introduction}

Methods of severe plastic deformation (SPD) have become very popular during the last years. Since the early works of Segal [1], who developed the process of equal channel angular extrusion (ECAE) for homogeneously refining microstructure of bulk metallic materials at cold working conditions. During ECAE a large uniform simple shear deformation is introduced into bulk billets without changing their cross section. This enables the application of multiple extrusions causing thereby a significant grain refinement and thus special physical and mechanical properties. Besides ECAE, other SPD-methods were developed, enhanced and investigated in detail by Valiev and co-workers [2] and many other researchers as shown by the immense number of SPD-related publications existing today [3].

However, the major part of the ECAE-related publications deals with easy to deform pure metals or solid-solution heat treated (SSHT) alloys as summarized in a recent review by [4]. ECAE for difficult-to-work metals is less reported since special precautions like elevated processing 
temperatures, channel intersection angles higher than $90^{\circ}$ or the application of a backpressure (BP) have to be applied to ensure homogeneous plastic deformation without cracking [4-9]. Since the materials get significantly strain hardened during SPD-processing, the grain refinement is mostly combined with a strong reduction in ductility $[3,4]$. Due to this considerable drawback, the areas of technical application for UFG materials are clearly limited, as a certain residual ductility is usually required. Recently a number of researchers focused on the combination of ECAP with an appropriate post-ECAE heat treatment in order to regain the ductility [10-17]. It was shown that a pre-ECAE solution heat treatment combined with post-ECAE annealing is very effective to achieve a good combination of strength and ductility for precipitation hardening $\mathrm{Al}$ alloys. This combination is attributed to the formation of very fine precipitates on the one hand, responsible for the high strength, and recovery processes enabling a certain strain hardening capability on the other hand. However, most of the investigations were conducted for SSHT conditions (except [17]). Less attention was paid to the influence of different pre- and post-ECAE heat treatments on the mechanical properties and the resulting workability.

The aim of the present study is to investigate the influence of different pre-ECAE heat treatments and processing temperatures on the workability and resulting mechanical properties of an $\mathrm{Al}-\mathrm{Cu}$ alloy produced by powder metallurgy (PM). Post-ECAE aging is performed for promising conditions to study their thermal stability and the resulting mechanical properties.

\section{Experimental}

The starting material was a commercial pre-alloyed spherical powder $(<100 \mu \mathrm{m})$ containing $4.1 \% \mathrm{Cu}-0.7 \% \mathrm{Mg}-0.8 \% \mathrm{Mn}-0.1 \% \mathrm{Si}-0.2 \% \mathrm{Fe}-$ blance $\mathrm{Al}$ in weight percent (equivalent to AA2017 with lowered Si-content). The powder was initially cold isostatically pressed at $\sim 200 \mathrm{MPa}$ into cylinders to $\sim 80 \%$ theoretical density and subsequently degassed for $4 \mathrm{~h}$ in a nitrogen atmosphere. Compaction was performed by direct extrusion at $\sim 390^{\circ} \mathrm{C}$ with a ram speed of $60 \mathrm{~mm} \cdot \mathrm{min}^{-1}$. The applied extrusion ratio of 22:1 ensured full densification for the final bars having a cross section of

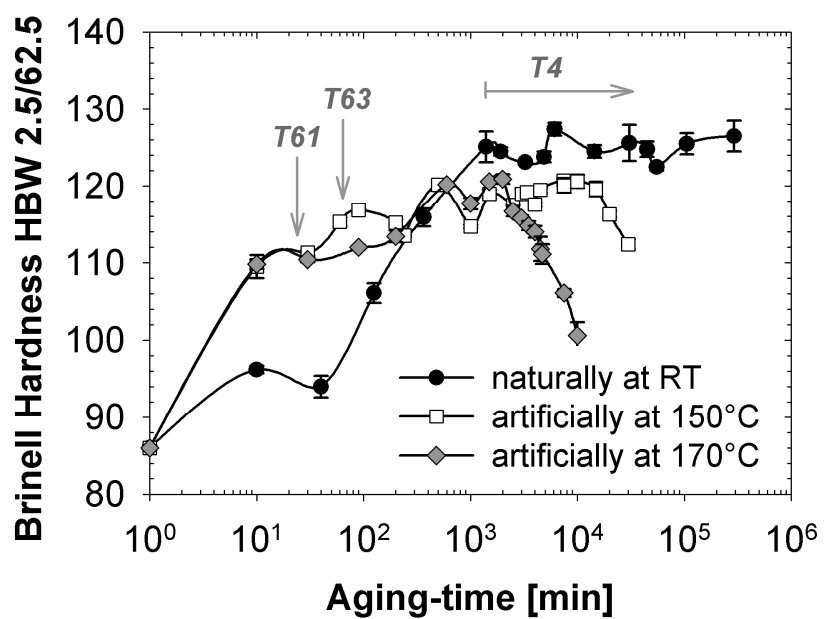
$15 \times 15 \mathrm{~mm}^{2}$. Subsequently the material was $\mathrm{SSHT}$ at $505^{\circ} \mathrm{C}$ for $60 \mathrm{~min}$, water quenched (WQ) and then naturally aged for about one month. This substantially stable condition is referred to as T4 hereafter and serves as reference. Figure 1 displays the aging plots for the investigated material for natural aging at RT and for artificial aging at $150^{\circ} \mathrm{C}$ and $170^{\circ} \mathrm{C}$.

Fig. 1: Aging plots for PM-AA2017 after SSHT at $505^{\circ} \mathrm{C}$ for $60 \mathrm{~min}$ followed by WQ.

Multi-pass ECAE processing was performed in a heatable die-set with an intersection angle of $90^{\circ}$, resulting in an equivalent plastic strain of $\sim 1.15$ per extrusion. The friction conditions inside the tooling are optimized by two movable walls in the inlet channel and a moving bottom slider in the outlet channel [18]. The ECA-extrusions were performed at $25 \mathrm{~mm} \cdot \mathrm{min}^{-1}$, following route $\mathrm{E}$ [19]. To ensure homogeneous deformation and to prevent from cracking, controlled BPs up to $300 \mathrm{MPa}$ were applied via the moving bottom slider. It is important to consider that an additional BP is induced by the frictional force at the slider bed.

Table 1 summarizes the routes and the corresponding parameters of the applied thermomechanical treatments (TMT). Exemplary designations are given as well. Excluding the time for heating $(\sim 10 \mathrm{~min})$, the time on temperature during ECAE was $\sim 5 \mathrm{~min}$ per pass. After processing at elevated temperatures, the billets were WQ. For the material processed in the SSHT-WQ condition 
below the conventional aging temperature, it is assumed that a natural aging process has occurred before tensile testing ( $\sim 1$ month later $)$.

Table 1: Parameters for the TMT-routes and designations for the PM-AA2017.

\begin{tabular}{ccc}
\hline Route & Parameters & Exemplary Designation \\
\hline TMT-1 & $\mathrm{T} 4 \rightarrow$ ECAE at $180^{\circ} \mathrm{C}, 220^{\circ} \mathrm{C}$ or $260^{\circ} \mathrm{C}$ up to $\mathrm{N}=8$ & T4 8E-220 ${ }^{\circ} \mathrm{C}$ \\
\hline TMT-2 & WQ $\rightarrow$ ECAE at RT up to $\mathrm{N}=2$ & WQ 1E-RT \\
\hline TMT-3 & WQ $1 \mathrm{E}-100^{\circ} \mathrm{C} \rightarrow$ aging at $140^{\circ} \mathrm{C}$ or $170^{\circ} \mathrm{C}$ & WQ 1E-100 $\mathrm{C}+140^{\circ} \mathrm{C}-15 \mathrm{~min}$ \\
\hline TMT-4 & $\begin{array}{r}\text { WQ } \rightarrow \text { artificial aging at } 170^{\circ} \mathrm{C} \text { for } 20 \text { min }(\mathrm{T} 61) \text { or } 60 \text { min } \\
(\mathrm{T} 63) \rightarrow \text { ECAE at } 160^{\circ} \mathrm{C} \text { or } 180^{\circ} \mathrm{C} \text { up to } \mathrm{N}=2\end{array}$ & T61 2E-160 $\mathrm{C}$ \\
\hline \hline
\end{tabular}

Brinell hardness measurements (according to EN ISO 6506-1) were performed in the section perpendicular to the direction of extrusion of the billets. For the characterisation of strength and ductility cylindrical tensile specimens with an aspect ratio of the gauge length of three were machined from the billets in the direction of extrusion. Quasi-static tensile tests were operated according DIN EN 10002 in a conventional testing machine from Zwick-Roell at constant crosshead speed with an initial strain rate of $10^{-3} \mathrm{~s}^{-1}$ at room temperature (RT). At least three parallel tests were performed. Typical curves are presented for the evaluation of the stress-strain behaviour. The yield stress at $0.2 \%$ plastic strain (YS), the ultimate tensile stress (UTS), the uniform elongation (UEL) and the elongation to failure (EL) are compared. Investigations of the microstructure were performed in the flow plane parallel to the direction of extrusion by transmission electron microscopy (TEM) with a Jeol-2010 from HITACHI operated at $200 \mathrm{kV}$. TEM-samples were mechanically ground to $\sim 60 \mu \mathrm{m}$ and further twin-jet thinned in the A2 electrolyte from Struers at $-30^{\circ} \mathrm{C}$.

\section{Results and discussion}

Figure 2a illustrates the stress-strain curves of the investigated PM-AA2017 after TMT route 1, wherein the processing was performed in the $\mathrm{T} 4$ condition at elevated temperatures between $180^{\circ} \mathrm{C}$ and $260^{\circ} \mathrm{C}$. The mechanical properties of the reference material are as follows: YS $=370 \mathrm{MPa}$, $\mathrm{UTS}=510 \mathrm{MPa}, \mathrm{UEL}=19.0 \%$ and $\mathrm{EL}=20.8 \%$. It can be seen clearly, that besides the processing temperature itself, the cumulative time at elevated temperature, determined by the number of extrusions $(\mathrm{N})$, has a significant influence on the resulting properties.

The application of temperatures above $220^{\circ} \mathrm{C}$ enabled the processing without additional BP, but significantly decreased the strength. The largest decrease of $\sim 50 \%$ was measured by the material that was processed for $\mathrm{N}=8$ at $260^{\circ} \mathrm{C}$ (exposed the longest time at the highest applied temperature).
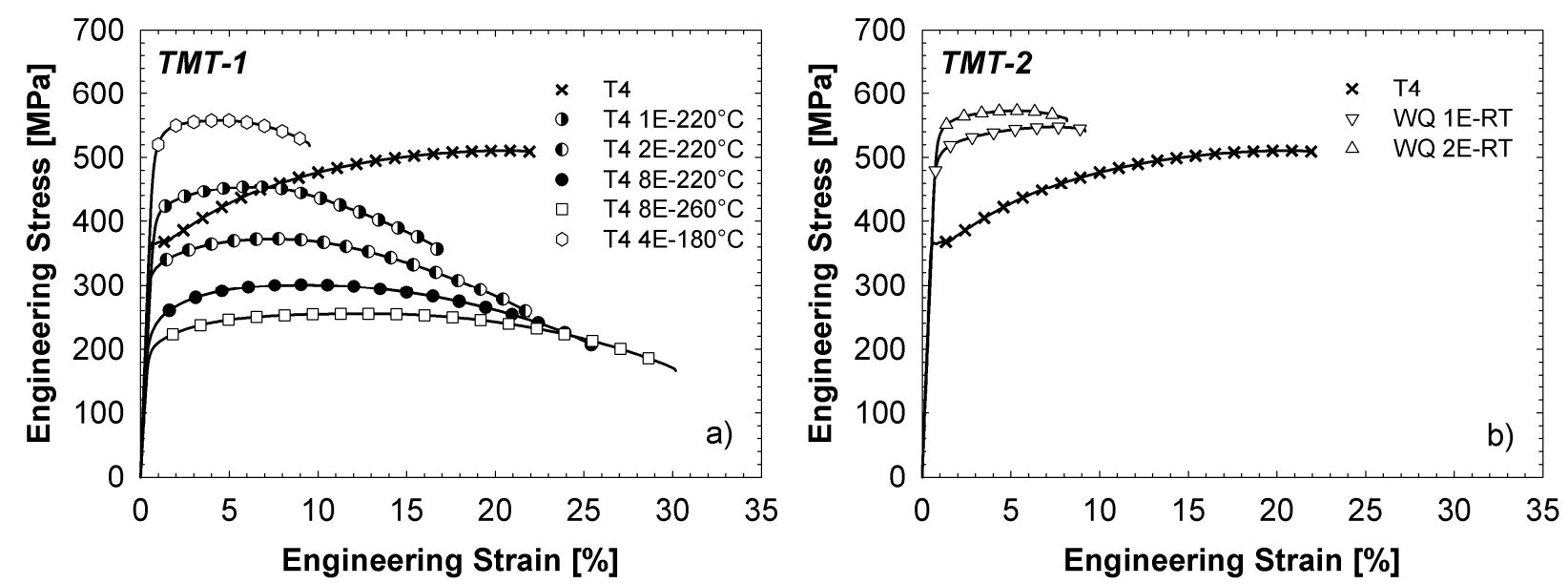

Fig. 2: Stress-strain curves for PM-AA2017 after a) TMT-1 and b) TMT-2. 
This behaviour is attributed to dynamic precipitation activity as well as to recovery which is effectively accelerated above the conventional aging temperature for $\mathrm{Al}-\mathrm{Cu}$ alloys of $\sim 150^{\circ} \mathrm{C}[20]$. The assumption is supported by the stress-strain curve of the condition processed at $180^{\circ} \mathrm{C}$, where thermal activated effects are retarded. At this temperature, sound billets could be produced up to $\mathrm{N}=4$ by the application of BP. This condition shows an increase of YS and UTS of $\sim 35 \%$ and $\sim 10 \%$ respectively, combined with a reasonable EL of $7.8 \%$. In order to introduce the deformation prior to aging, RT-processing was conducted according to TMT route 2 . Therefore maximum BP was necessary to prevent the billets from cracking. This is in accordance with the results on WQAA2024 [14, 17] and WQ-AA2124 [21] where cracks were reported already during the first ECAextrusion below $\sim 150^{\circ} \mathrm{C}$ without additional BP and at RT with $75 \mathrm{MPa}$ additional BP, respectively. According to Figure $2 \mathrm{~b}$ a significant increase in strength is achieved after processing following TMT-2, while YS benefits in particular (YS $+45 \%$, UTS $+15 \%$ for WQ 2E-RT). The ductility is comparable with that of $\mathrm{T} 44 \mathrm{E}-180^{\circ} \mathrm{C}$ in TMT- 1 . Thus, processing following TMT-2 is more efficient if compared to TMT-1.

To investigate the influence of a post-ECAE heat treatment on the pre-ECAE SSHT condition, instantaneous aging was performed after ECAE-processing following TMT route 3 . In order to reduce the loading of the ECA-die to a moderate level, the processing temperature was elevated to $100^{\circ} \mathrm{C}$, enabling a reduction of the BP. Figure 3 summarizes the development of the hardness and the mechanical properties as a function of time for aging at $140^{\circ} \mathrm{C}$ and $170^{\circ} \mathrm{C}$.
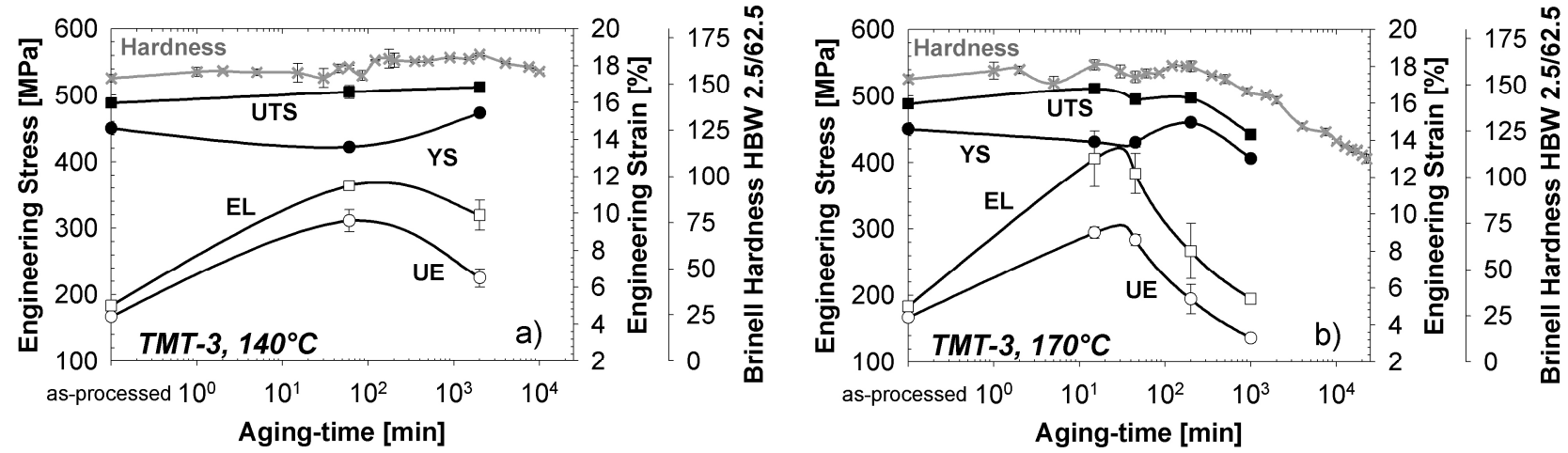

Fig. 3: Aging plots for TMT-3 (WQ $1 \mathrm{E}-100^{\circ} \mathrm{C}$ ) during post ECA-aging at a) $140^{\circ} \mathrm{C}$ and b) $170^{\circ} \mathrm{C}$.

A comparison of the as-processed condition $\left(\mathrm{N}=1\right.$ at $\left.100^{\circ} \mathrm{C}\right)$ with the corresponding condition in TMT-2 ( $\mathrm{N}=1$ at $\mathrm{RT})$, reveals yet again the negative effect of in-situ precipitation on the resulting mechanical properties, as both, a lower strength and ductility, is achieved for the higher processing temperature. Nevertheless, a considerable effect of the mechanical properties can be observed. A comparison of strength and hardness yields good agreement. Both parameters follow an almost horizontal trend before softening begins at $\sim 2000 \mathrm{~min}$ at $140^{\circ} \mathrm{C}$ and at $\sim 200 \mathrm{~min}$ at $170^{\circ} \mathrm{C}$. Compared to the aging of the unprocessed material the hardness drops already after a rather short time (cp. Fig. 1), suggesting an acceleration of aging kinetics due to ECAE. Corresponding to the results reported in [10], for AA6060 the ductility increases during the beginning of aging and decreases again beyond an optimum aging time. Accordingly a maximum EL of $13 \%$ is reached after aging for only $15 \mathrm{~min}$ at $170^{\circ} \mathrm{C}$. The corresponding YS is increased for $\sim 15 \%$ while UTS is maintained if compared to T4.

Cheng et al. reported for a AA2024 [22] that workability at cryogenic temperatures benefits from remnant precipitates that were partially dissolved during an incomplete SSHT. This is in accordance with Lapovok et al. [8] who also reported better RT-ECAE workability for annealed AA2124 compared to the SSHT condition. According to Figure 4 the SSHT PM-AA2017 was artificially aged at $170^{\circ} \mathrm{C}$ for $20 \mathrm{~min}$ (T61) and $60 \mathrm{~min}$ (T63) prior to ECAE in order to improve the workability. These under-aged conditions were processed according to TMT route 4 at temperatures close to the pre-aging temperature. Despite the insignificant difference in hardness (see Fig. 1), the 
T61 condition reveals better workability compared to the T63, enabling the application of medium $\mathrm{BP}$ to ensure homogeneous plastic deformation up to $\mathrm{N}=2$. ECA-processing at $180^{\circ} \mathrm{C}$ yields an

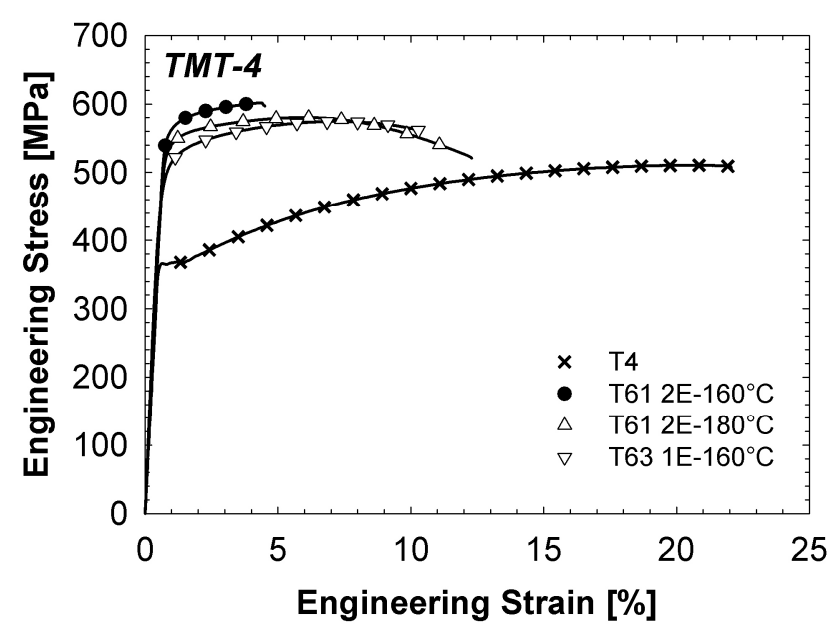
increase of YS and UTS of $\sim 40 \%$ and $\sim 15 \%$ respectively, combined with a remarkably EL of $11.6 \%$. According to the former results, the material extruded at lower temperature reveals higher strength but also reduced ductility. Due to the worse workability of the T63 condition, only one extrusion at $160^{\circ} \mathrm{C}$ with maximum BP could be performed. The stress-strain curve of this condition reveals qualitatively the same characteristics as the T61-condition after $\mathrm{N}=2$ processed at $180^{\circ} \mathrm{C}$.

Fig.4: Stress-strain curves for PM-AA2017 after TMT route 4.

To correlate the mechanical behaviour with the microstructure, systematic TEM-investigations were performed. The average grain size of the T4 condition is shown in Figure $5 \mathrm{a}$ is $\sim 5 \mu \mathrm{m}$. Besides a very low dislocation density, rod-shaped and spherical particles are present which were identified as remnant $\mathrm{T}$-phase precipitates in [23]. Figure $2 \mathrm{~b}$ and $\mathrm{c}$ show representative micrographs for the condition $\mathrm{T} 612 \mathrm{E}-160^{\circ} \mathrm{C}$ at lower and higher magnification. It is visible that dislocations were effectively accumulated during ECA-processing, forming a cellular substructure. The selected area electron diffraction pattern (SAED) still shows concentrations revealing the coexistence of both low- and high-angle grain boundaries. This suggests that the formation of a truly homogeneous ultrafine-grained microstructure has not been completed. Nevertheless, ultrafine grains with an average size of $\sim 300 \mathrm{~nm}$ are formed in some regions. As shown in Figure $5 \mathrm{c}$ the grain boundaries in these areas are mostly of high-angle type, indicated by the clearness of their appearance.

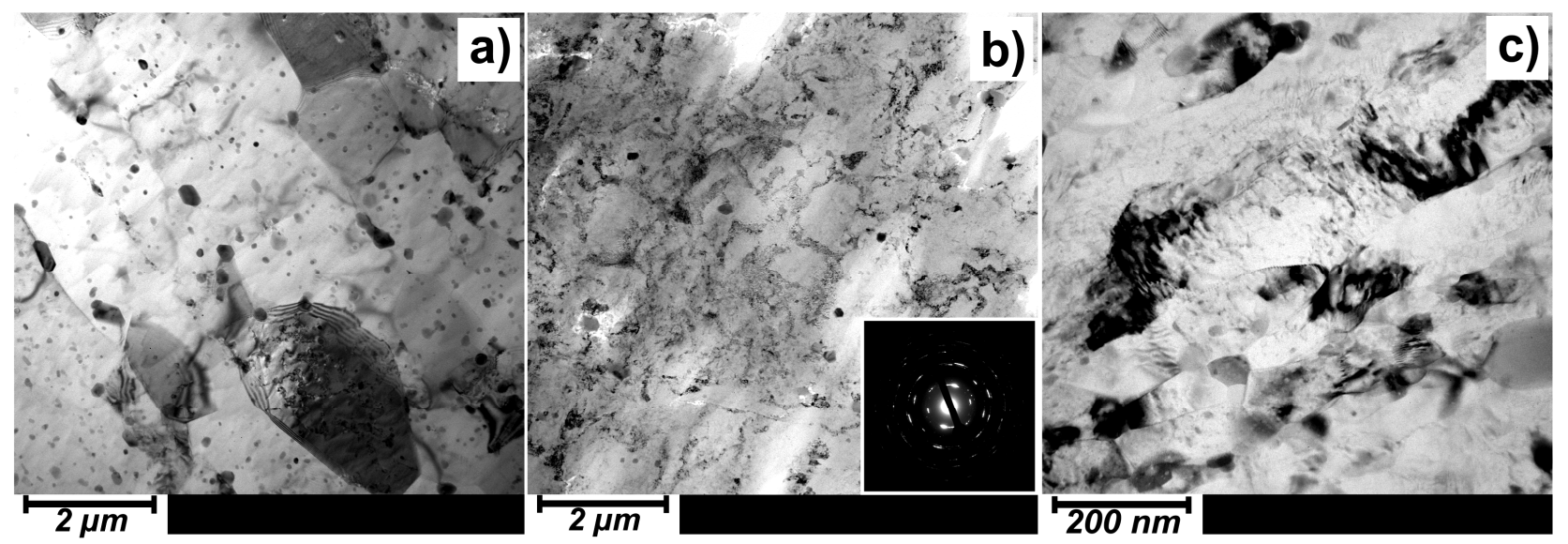

Fig. 5: Bright-field TEM-micrographs for the PM-AA2017 a) T4 , b) TMT-4, T61 2E-160 ${ }^{\circ} \mathrm{C}$ overview with SAED, c) TMT-4, T61 $2 \mathrm{E}-160^{\circ} \mathrm{C}$ with higher magnification

\section{Summary and Conclusions}

In this study different thermo-mechanical treatments were applied on PM-AA2017 to with respect to the workability, in order to achieve a reasonable combination of strength and ductility.

1. ECA-processing of the naturally aged condition above $220^{\circ} \mathrm{C}$ does not necessitate $\mathrm{BP}$ and yields a continuous decrease of strength with increasing extrusion numbers. Contrary ECAextrusions at $180^{\circ} \mathrm{C}$ requires BP and gives increases in strength and reasonable ductility. 
2. RT-processing of the pre-ECAE SSHT condition is possible up to $\mathrm{N}=2$ by the application of maximum BP. A significant increase in strength is achieved, while YS benefits in particular.

3. Post-ECA aging of pre-ECAE SSHT conditions increases the ductility to notable values while retaining the strength.

4. A combination of high strength and moderate ductility was achieved by ECA-processing of an under-aged condition, enabling the application of medium BP.

\section{Acknowledgements}

The authors gratefully acknowledge the Deutsche Forschungsgemeinschaft (DFG) for supporting this work carried out within the framework of Sonderforschungsbereich 692 (Collaborative Research Center A1, A2).

\section{References}

[1] V. M. Segal, A. E. Reznikov, A. E. Drobyshevskiy, V. I. Kopylov, Russ. Metall. (english translation) (1981) 7.

[2] R. Z. Valiev, R. K. Islamgaliev, I. V. Alexandrov, Progress in Materials Science 45 (2000) $103-$ 189.

[3] Information on http://www.nanospd.org/, in: 2008.

[4] R. Z. Valiev, T. G. Langdon, Progress in Materials Science 51 (2006) 881-981.

[5] V. N. Chuvil'deev, T. G. Nieh, M. Y. Gryaznov, V. I. Kopylov, A. N. Sysoev, Journal of Alloys and Compounds 378 (2004) 253-257.

[6] I. Karaman, G. G. Yapici, Y. I. Chumlyakov, I. V. Kireeva, Materials Science and Engineering: A $410-411$ (2005) 243-247.

[7] L. J. Kecskes, K. C. Cho, R. J. Dowding, B. E. Schuster, R. Z. Valiev, Q. Wei, Materials Science and Engineering: A 467 (2007) 33-43.

[8] R. Lapovok, C. Loader, F. H. D. Torre, S. L. Semiatin, Materials Science and Engineering: A 425 (2006) 36-46.

[9] I. P. Semenova, G. I. Raab, L. R. Saitova, R. Z. Valiev, Materials Science and Engineering A 387-389 (2004) 805-808.

[10] M. Hockauf, L. W. Meyer, B. Zillmann, M. Hietschold, S. Schulze, L. Krüger, Materials Science and Engineering: A Accepted for publication.

[11] Z. Horita, Ohashi K, T. Fujita, K. Kaneko, T. G. Langdon, Advanced Materials 17 (2005) 1599-1602.

[12] J. K. Kim, H. G. Jeong, S. I. Hong, Y. S. Kim, W. J. Kim, Scripta Materialia 45 (2001) 901907.

[13] J. K. Kim, H. K. Kim, J. W. Park, W. J. Kim, Scripta Materialia 53 (2005) 1207-1211.

[14] W. J. Kim, C. S. Chung, D. S. Ma, S. I. Hong, H. K. Kim, Scripta Materialia 49 (2003) 333 338.

[15] W. J. Kim, J. K. Kim, H. K. Kim, J. W. Park, Y. H. Jeong, Journal of Alloys and Compounds In Press, Corrected Proof (in press).

[16] W. J. Kim, J. Y. Wang, Materials Science and Engineering: A 464 (2007) 23-27.

[17] J. Mao, S. B. Kang, J. O. Park, Journal of Materials Processing Technology 159 (2005) 314320.

[18] V. M. Segal, Materials Science and Engineering A 386 (2004) 269-276.

[19] R. E. Barber, T. Dudo, P. B. Yasskin, K. T. Hartwig, Scripta Materialia 51 (2004) 373-377.

[20] E. A. Brandes, G. B. Brook, Smithells Light Metals Handbook (1998).

[21] R. Y. Lapovok, The positive role of back-pressure in equal channel angular extrusion, in: Materials Science Forum, vol 503-504, Fukuoka, 2006, pp. 37-44.

[22] S. Cheng, Y. H. Zhao, Y. T. Zhu, E. Ma, Acta Materialia 55 (2007) 5822-5832.

[23] S. C. Wang, M. J. Starink, International Materials Reviews 50 (2005) 193-215. 